\title{
KANTS MORALPHILOSOPHIE UND IHRE KOMMENTARISCHE INTERPRETATION
}

Ein Interview mit Dieter Schönecker

von Robinson dos Santos (UFPEL)

\section{Apresentação}

Dieter Schönecker (1965) é um dos mais renomados pesquisadores jovens da filosofia de Kant na atualidade. Realizou seus estudos de doutorado em Filosofia, Ciência Comparada da Religião e Literatura na Universidade de Bonn, período no qual foi bolsista (visiting fellow) na Universidade de Yale (USA). Foi professor assistente nas Universidades de Bonn e Halle-Wittenberg na Alemanha entre os anos de 1999 e 2002. Retornou aos Estados Unidos como Professor Associado do Stonehill College permanecendo lá de 2003 até 2005. Desde 2006 é Professor de Filosofia Prática na Universidade de Siegen, Alemanha.

No ano de 2008, Dieter Schönecker esteve em Porto Alegre, como conferencista convidado, no IV Congresso Kant da Sociedade Kant Brasileira. Entre seus principais trabalhos publicados encontramos: Kant: Grundlegung III. Die Deduktion des kategorischen Imperativs (1999); Kants "Grundlegung zur Metaphysik der Sitten". Ein einführender Kommentar, escrito em parceria com Allen W. Wood (2002) - título que já está na terceira edição; Kants Begriff transzendentaler und praktischer Freiheit. Eine entwicklungsgeschichtliche Studie (2005), pertencente à série Kant-Studien Ergänzungshefte. Além destas é co-autor e organizador de: Moralische Motivation. Kant und die Alternativen (2006); Groundwork for the
Metaphysics of Morals (2006); Der moralische Status menschlicher Embryonen. Argumente pro und contra Spezies-, Kontinuums-, Identitäts- und Potentialitätsargument (2003); Kant verstehen/Understanding Kant. Über die Interpretation philosophischer Texte (2001).

Além de exercer as atividades de ensino e pesquisa, Schönecker coordena o Centro para Interpretações Comentadoras da obra de Kant [Zentrum für Kommentarische Interpretationen zu Kant], junto a Universidade de Siegen, que promove anualmente duas modalidades de encontros para estudos e intercâmbio de pesquisadores: o Siegener Kant Kurs volta-se aos estudantes em fase de mestrado e doutorado e o Siegener Kant Tagung é um congresso para pesquisadores cuja titulação seja, no mínimo, o doutorado completo.

Nesta entrevista - que o Professor Schönecker gentilmente nos concedeu - ele nos conta um pouco de sua trajetória intelectual e formativa (1), bem como nos esclarece alguns termos que emprega no trabalho com as obras de Kant, tais como interpretação comentadora [kommentarische Interpretation] e esquecimento do texto [Textvergessenheit] (2).Em seguida explica como surgiu a idéia de fundar o Zentrum für kommentarische Interpretationen zu Kant e apresenta o(s) objetivo(s) do mesmo (3). Além destes aspectos Schönecker expõe seu 
ponto de vista sobre algumas questões (sempre repetidas) como as acusações de rigorismo e formalismo na ética de Kant (4) e, em particular, a interpretação/recepção de Kant pela ética do discurso (5). Por fim, tece considerações sobre a pertinência da ética kantiana no contexto de problemas atuais (6).

\section{Interview}

ethic@ - Herr Professor Schönecker, wieso haben Sie Philosophie als Fachstudium gewählt? Wie ist Ihre Berührung mit Kants Philosophie entstanden? Können Sie uns etwas über Ihre Akademische Laufbahn erzählen?

DIETER SCHÖNECKER - Mein Interesse an der Philosophie wurde durch meinen Zweifel an der Existenz Gottes geweckt. Dieser Zweifel hat mich als Jugendlicher sehr erschüttert, und eigentlich ist er das, was mich bis heute philosophisch nährt. Ich habe dann Philosophie bereits in der Schule gelernt und nach dem Abitur das Studium der Philosophie, Deutschen Literatur und der Vergleichenden Religionswissenschaft an der Universität Bonn aufgenommen. Während meines Studiums habe ich als Gaststudent ein Jahr an der University of Massachusetts at Amherst verbracht, was insofern prägend war, als ich erst dort wirklich meine Vorliebe für die analytische Philosophie entdeckt habe (aus der Vorliebe ist im Laufe der Jahre aber eher eine Hassliebe geworden). Nach dem Magister habe ich dann in Bonn bei Hans Michael Baumgartner mit einer Arbeit über die Deduktion des kategorischen Imperatives im Dritten Abschnitt von Kants „Grundlegung zur Metaphysik der Sitten“ (GMS) promoviert.
Allerdings war diese Betreuung mehr aus der Not geboren, da mir sonst in Bonn niemand dafür geeignet schien; ich war also kein Schüler Baumgartners im engeren Sinne und hatte mit ihm über Kant und meine Arbeit so gut wie keinen Austausch.

In Bonn war es unvermeidlich, mit Kant in Berührung zu kommen; viele Dozenten haben über ihn geforscht und gelehrt (neben Baumgartner auch z.B. Baumanns, Marx, Oberer, Simon). Im Rahmen einer Hausarbeit über den Dritten Abschnitt von Kants GMS wurde mir noch einmal deutlich, was meine interpretatorische Arbeit bis heute bestimmt: dass nämlich nicht genau gelesen wird. Ich habe diese Arbeit dann zur Magisterarbeit weiterentwickelt und schließlich zur Dissertation.

Nach der Promotion war ich für knapp zwei Jahre als Visiting Fellow an der Yale University, wo ich mit Allen Wood zusammengearbeitet habe (daraus ist ein Kommentar zur GMS entstanden); dafür bin ich sehr dankbar, weil ich durch ihn die Wichtigkeit auch der genuinen Sachanalyse eingesehen habe (die allerdings auf der Textanalyse beruhen muss). Nach drei weiteren Jahren als wissenschaftlicher Assistent an der Universität Halle-Wittenberg bin ich dann wieder zurück in die USA gegangen, wo ich als Professor für Philosophie am Stonehill College in der Nähe von Boston unterrichtet habe. Seit Januar 2006 bin ich Professor für Praktische Philosophie an der Universität Siegen.

ethic@- Können Sie uns erklären, was eine kommentarische Interpretation ist? Und was bedeutet der Ausdruck "Textvergessenheit", 
den man häufiger bei Ihnen findet?

DIETER SCHÖNECKER - Die Frage nach dem Wesen der kommentarischen Interpretation bringt mich immer in die Verlegenheit, etwas erklären oder sogar definieren zu müssen, das, wenn man es abstrakt darstellt, nur klingt wie ein leerer Allgemeinplatz. Einen philosophischen Text kommentarisch zu interpretieren heißt nichts anderes als: Genau zu lesen und die Frage nach der Bedeutung von der Frage nach der Wahrheit zu unterscheiden. Und da natürlich niemand bestreitet, dass man genau lesen muss, ist zumindest dieses zentrale Element der kommentarischen Interpretation auf eine scheinbare triviale Weise so unbestreitbar, dass man sich kaum traut, es zu benennen. Es würde ja niemand sagen: Nein, Herr Schönecker, das bestreite ich, ich lese mit Absicht ungenau!

Lassen Sie mich für einen Augenblick noch einmal zurückschauen: Schon im ersten Semester hatte ich nach dem Besuch philosophischer Lektüreseminare den starken Eindruck, dass niemand genau liest: Es wurde sehr schnell, zu schnell, philosophiert in dem Sinne, dass man über die Sache, die man anhand des Textes rasch zur Hand zu haben meinte, diskutierte und nicht über den Text, in dem allein sich die Sache - ein Argument, eine These, eine Theorie - ja überhaupt nur befindet. (Das ging mir übrigens auch in der Literaturwissenschaft so: Zwar geht es da nicht um Argumente; aber auch bei der Interpretation eines Gedichts wie „Willkommen und Abschied“"von Goethe sollte man eigentlich erwarten dürfen, das Fragen der Form gestellt werden wie ,was bedeutet das?“, oder „Warum steht da x und nicht y?“. Doch das fand nicht statt. Statt dessen schwafelte man über alle möglich Theorien, etwa der „Entdeckung des Ich im Sturm und Drang“ oder so etwas.) Mein Eindruck als Student war ganz einfach der, dass die Lektüre wirklich viel genauer sein kann; und verstärkt wurde dieses Erlebnis auch durch die dekonstruktivistischen Lesarten in der Schule des Bonner Philosophen Josef Simon, bei dem es kaum noch einen geordneten Textbezug gab.

Meine These lautet: Es gibt mit ganz wenigen Ausnahmen keine wirklich systematische, textorientierte und historiographische Kant-Forschung. Die Tatsache, dass es eine ungeheuer große Zahl von Veröffentlichungen zu Kant gibt, ändert daran nichts. Natürlich sind, rein quantitativ betrachtet, einzelne Bereiche von Kants Werk weniger bearbeitet als andere. Aber nicht ein einziges Werk der Kantischen Philosophie und erst recht kein einzelnes Gebiet dieser Philosophie ist wirklich grundlegend erforscht; hier gibt es noch sehr viel zu entdecken. Ich habe dieses Phänomen an anderer Stelle als Textvergessenheit bezeichnet und ausführlicher behandelt. Der Kerngedanke ist einfach: Es ist eine Sache, einen Text $\mathrm{zu}$ verstehen und eine andere, ihn $\mathrm{zu}$ bewerten (wenn auch Bedeutungssuche ohne Wahrheitsüberlegungen in der Regel nicht möglich ist). Sinn und Zweck der Kant-Forschung ist es also, die Texte Kants (besser) zu verstehen; es ist nicht ihre (primäre) Aufgabe, den Inhalt der Kantischen Philosophie weiterzuentwickeln oder $\mathrm{zu}$ kritisieren (was man weiterentwickeln oder kritisieren will, muss man eben zunächst einmal verstehen). Dieser Unterschied wird nicht begriffen oder jedenfalls nicht respektiert, und auch aus diesem Grund mangelt es in der Kant-Literatur 
an wirklich textnahen Analysen. Wer einen Text verstehen will, muß bereit sein, sich auf allerkleinste Details und mikroskopische Feinheiten, zugleich aber auf kontextuelle und entwicklungsgeschichtliche Zusammenhänge einzulassen. Doch wer ist dazu bereit? Wer wäre in der Lage, auch nur einen einzigen Kommentar zu benennen, der etwa die „Kritik der reinen Vernunft" wirklich Satz für Satz und Wort für Wort auch nur zu verstehen bemüht wäre? Um keine Mißverständnisse aufkommen zu lassen: Ich rede keiner blinden Kantphilologie das Wort. Wer sinnvoll Philosophiehistorie betreiben will, sollte zugleich auf dem neuesten Stand der philosophischen Forschung sein, und natürlich dürfen Philosophiehistoriker auch philosophische Aussagen machen (als Philosophen geht es uns natürlich letztlich um solche Aussagen). Aber die Aussage „Kant hält Raum und Zeit für bloße Anschauungsformen“" hat doch offenkundig einen völlig anderen Status als die Aussage „Kant ist der Auffassung, dass Raum und Zeit bloße Anschauungsformen sind, und er hat Recht damit".

Sehr wichtig ist mir noch folgendes: Ich meine mit der kommentarischen Interpretation nicht, dass ich, wenn ich zum Beispiel ein Buch über Kants Kritik der reinen Vernunft schreibe, dann Satz für Satz den Text kommentiere. Ich muss natürlich auf jeden Satz eingehen, jeder Satz muss irgendwo Erwähnung finden. Aber es ist absurd $\mathrm{zu}$ glauben, man könnte einen sinnvollen Kommentar zu irgendeinem Buch schreiben, wenn man in der Darstellung wirklich Satz für Satz vorgeht. Kant schreibt vielleicht in irgendeinem Buch im ersten Kapitel etwas über Freiheit, und er schreibt dann wieder etwas über Freiheit im fünften Kapitel.
Wenn ich jetzt einen Kommentar schriebe, der wirklich Satz für Satz vorginge, dann würde ich am Anfang etwas über Freiheit sagen, dann lange gar nichts darüber sagen, und dann wieder etwas über Freiheit sagen im fünften Kapitel. Aber ich muss im Kommentar natürlich diese Stellen zusammenbringen. Und dieses Zusammenbringen und dieses genaue Lesen, das ist das, was ich unter „kommentarisch“ verstehe .

ethic@ - Wie sind Sie dazu gekommen das Zentrum für kommentarische Interpretation zu Kant zu gründen?

DIETER SCHÖNECKER - Die Idee für ein Zentrum für kommentarische Interpretation zu Kant (www.zetkik.de) hatte ich schon länger, aber erst der Ruf an die Universität Siegen hat es mir ermöglicht, sie auch umzusetzen. Das ZetKIK will, allgemein gesprochen, kommentarische Interpretation realisieren und bekannt machen. Das geschieht durch die Förderung von Einzelstudien (Dissertationen usw.), aber vor allem auch durch die Siegener Kant-TagungenunddieSiegenerKant-Kurse(für den wissenschaftlichen Nachwuchs), die jeweils einmal im Jahr stattfinden. Dabei versuchen wir, in jeweils zwei Tagen eine kleine Textpassage (z.B. Kants Hinweise auf Kopernikus in der $\mathrm{KrV}$ ) gemeinsam zu interpretieren, und zwar ganz ohne Vorträge; wir sitzen einfach nur am runden Tisch des ZetKIK und arbeiten ganz eng (eben kommentarisch) am Text. Neben dem Austausch hat das den großen Vorteil, dass die Kollegen und Nachwuchsforscher konkret erleben können, was eigentlich eine kommentarische Interpretation auszeichnet. Es 
ist, wie gesagt, einnaheliegender (und tatsächlich oft wiederholter) Einwand, dass die Forderung nach genauem Lesen nicht sonderlich originell wäre, da ja ohnehin alle wüßten, was genaues Lesen sei und beinhalte. Die Erwiderung darauf kann nur der Verweis auf die echte Arbeit und Leistung kommentarischer Interpretation sein. (Ich darf daher auch alle einladen, sich für diese Veranstaltungen $\mathrm{zu}$ bewerben. Bisher hatten wir das große Glück, von der Thyssen-Stiftung und der Deutschen Forschungsgemeinschaft gefördert zu werden, so dass wir Reisestipendien vergeben konnten.)

Ein weiterer Aspekt des ZetKIK ist das gemeinsame Forschen. Denn ein Problem der Kantforschung ist nicht nur die Textvergessenheit, sondern auch die Tatsache, dass von einem echten Stand der Kantforschung auch deswegen keine Rede sein kann, weil ein echter Forschungsstand nur möglich ist, wenn neuere Forschung auf bereits geleisteter aufbaut. Schon 1881 beklagte bekanntlich Vaihinger, dass niemand mehr in der Lage sei, die Literatur $\mathrm{zu}$ überschauen. Und in der Tat: Es ist für Einzelne schlichtweg unmöglich, die Literatur sinnvoll zu verarbeiten. Erschwerend kommt hinzu, dass die Literatur zu Kant in mehreren europäischen Grundsprachen verfasst ist. Üblicherweise werden nicht alle diese Sprachen beherrscht, und sobleiben Forschungsergebnisse wirkungslos, schlicht und einfach deshalb, weil sie aus sprachlichen Gründen gar nicht gelesen werden können. Diese Unübersichtlichkeit hat enorme Konsequenzen: Wir wissen nämlich nicht einmal, was bisher zu Kant überhaupt auch nur vorgeschlagen worden ist und welche Einzelinterpretationen, Beobachtungen und Lösungsvorschläge gemacht worden sind.
Niemand weiß, was bisher geleistet wurde. Das führt aber zwangsläufig dazu, dass Erkenntnisse überhaupt nicht wahrgenommen werden, verlorengehen und stets aufs neue vorgetragen werden; es wird gewissermaßen das Rad wieder und wieder erfunden, so etwas wie einen echten ,Forschungsstand' gibt es in der Kantforschung überhaupt nicht. Dass viele Autoren nicht einmal versuchen, zumindest neuere Literatur $\mathrm{zu}$ berücksichtigen und so tun, als gäbe es bestimmte Probleme und Lösungsvorschläge überhaupt nicht, steht auf einem anderen Blatt. Es ist übrigens ein Skandal, dass vor allem der englischsprachige Markt (vor allem der USA) die Kant-Szene beherrscht, obwohl viele dieser angeblichen Forscher nicht einmal der deutschen Sprache mächtig sind und in der Regel sehr textvergessen arbeiten. Die ganze Welt rezipiert und diskutiert die Beiträge der US-Philosophen, aber umgekehrt kommt dies so gut wie gar nicht vor. Nach meiner Erfahrung ist das übrigens in der der spanisch- und portugiesischsprachigen Kant-Szene ganz anders.

ethic@ - In Rahmen der Interpretationen von Kants Moralphilosophie gibt es viele „Gemeinsprüche“, die immer wieder benannt werden. Ein Beispiel davon ist der Rigorismus oder Formalismus Vorwurf. Ist die Moralphilosophie Kants im diesem Sinne korrekturbedürftig, oder ist das eine fehlerhafte Interpretation?

DIETER SCHÖNECKER - Diese Frage lässt sich nicht allgemein beantworten. Denn erstens muss ja klar sein, was genau etwa „Rigorismus“ oder „Formalismus“ bedeuten (oder die anderen Standardvorwürfe, wie etwa 
auch der, Kants Ethik sei „metapyhsiklastig“"). Und zweitens muss man auch differenzieren. So ist die vielleicht wirkmächtigste und auch umstrittenste Frage hinsichtlich des kategorischen Imperativs immer noch, wie genau überhaupt das (angebliche) Verfahren der Verallgemeinerung von Maximen bei Kant funktionieren soll (und ob es funktioniert); es gibt vermutlich sehr wenige Stellen aus der ethischen Weltliteratur, die so intensiv diskutiert wurden wie die berühmten Beispiele aus dem zweiten Abschnitt der GMS. Damit verbunden ist der prominent von Hegel und Scheler vorgetragene Vorwurf des (angeblichen) Formalismus, der allerdings zu einseitig auf die Universalisierungsformel fokussiert ist und dabei vergessen hat, dass es auch die Zweckan-sich-Formel gibt, die keineswegs über so etwas wie Universalisierung läuft; so hat Kant auch in der tugendethischen und postmodernen Kritik am ethischen Universalismus bevorzugt als Prügelknabe herhalten müssen. Der prominent von Schiller und später etwa auch von Schopenhauer vorgetragene Vorwurf, Kant habe kein angemessenes Verständnis moralisch relevanter Gefühle wie Menschenliebe, Freundschaft oder Mitleid und kenne nur das rigide Handeln aus Pflicht, ist bis heute nicht verstummt, wobei auch in diesem Punkt die Debatte stark durch die einseitige Konzentration auf die GMS verzerrt war und ist. Stärker systematisch orientiert (und interpretatorisch wohl weitgehend zutreffend), ist m.E. die Kritik an Kants (modern gesprochen: antikonsequentialistischem) Rigorismus bezüglich der absoluten Ausnahmslosigkeit ethischer Handlungstypengebote. Kant ist Rigorist, aber vielleicht ist das ja nicht verkehrt. ethic@ - Was ist ihre Meinung über die KantRezeption bei Interpretationen wie z.B. die Diskursethik?

DIETER SCHÖNECKER - Kant ist natürlich in vielfältiger Weise und von verschiedenen Schulen sowohl hinsichtlich seiner theoretischen wie auch hinsichtlich seiner praktischen Philosophie rezipiert worden. Die Antwort auf die Frage, wie nah solche Aneignungen noch an Kant sind, lässt sich nicht verallgemeinern. Um die Diskursethik aufzugreifen: Natürlich gibt es Gemeinsamkeiten zwischen Kants Ethik und der Diskursethik, wie etwa den Kognitivismus und den Universalismus und in gewisser Hinsicht auch den Formalismus, und Philosophen wie Karl-Otto Apel und Jürgen Habermas, besonders aber auch Apels Schüler Wolfgang Kuhlmann, haben solche Gemeinsamkeiten auch betont (wobei allerdings der Grundgedanke der transzendentalen Begründung und damit die Idee der Letztbegründung in der theoretischen Philosophie für die Transzendentalpragmatik m.E. wichtiger war als der eigentlich ethische Bereich). Aber was ist damit denn gesagt? Kognitivistisch und universalistisch sind viele Varianten in der Ethik, und formalistisch ist Kants Ethik nur begrenzt. Die Diskusethik hat also in der Tat einiges mit der Kantischen Ethik gemein, aber damit ist eben nicht viel gesagt. Die Kernidee der Diskursethik - bei der dann man allerdings wieder deutlich differenzieren muss, vor allem auch, was Letztbegründungsansprüche betrifft - besagt, das moralische Normen sich transzendentalpragmatisch aus den Bedingungen der Präsuppositionen von Sprache und Kommunikation begründen lassen. Dieser Gedanke findet sich bei Kant nicht (wenn 
es auch im Dritten Abschnitt der GMS ein Argument für die Freiheitsannahme gibt, das man transzendentalpragmatisch nennen könnte).

ethic@-Angesichts der neuen und komplexen Probleme im Bereich der Wissenschaft und der Ethik: Sind Sie der Meinung, dass neue Zeiten eine neue Ethik fordern? Oder, mit anderen Worten: Wie aktuell ist eigentlich Kants Moralphilosophie?

DIETER SCHÖNECKER - Das sind in Wahrheit ganz verschiedene Fragen. Zunächst: Ich glaube nicht, dass sich in der Ethik - genauso wenig wie in der Philosophie überhaupt - auf der grundsätzlichen Ebene etwas substantiell Neues sagen lässt. Zwar gibt es neue Begriffe (Kognitivismus, Realismus, Internalismsus usw.), und besonders auch die US-amerikanische Philosophie in der Analytischen Tradition ist in Hollywood-Manier sehr geschickt darin, die Diskussion durch Wortneuschöpfungen zu prägen. Doch hier gibt es nichts Neues unter der Sonne. In diesem Sinne ist Kants Moralphilosophie also genau so aktuell wie die anderen Haupttypen Philosophischer Ethik. Sie ist aber auch aktuell in dem Sinne, dass Ethiken Kantischen Typs (also nicht Kants Ethik als solche) ohne Zweifel - im Unterschied etwa, sagen wir, zur naturrechtlichen Tradition - zu den Ethiktypen gehören, die weit verbreitet sind und intensiv diskutiert werden (man denke etwa ganz aktuell an die neuen Arbeiten von Derek Parfit, die im Internet kursieren). Anders verhält es sich mit der sogenannten Angewandten Ethik. Da erwachsen durch den Fortschritt in den Wissenschaften konkrete Fragen, die es vorher einfach nicht gegeben hat, weil es das Wissen oder sogar die Dinge selbst nicht gab; man denke etwa an die Frage nach dem moralischen Status induzierter pluripotenter Stammzellen. In Bezug auf Deutschland kann man übrigens feststellen, dass faktisch die richterliche und fachwissenschaftliche Auslegung unseres Grundgesetzes ohne Kants Ethik undenkbar ist; und auch bei den Fragen der Angewandten Ethik, die in den letzten Jahren hierzulande die Diskussion bestimmt haben z.B. Stammzellforschung, Folter, Sterbehilfe wird immer wieder auf Kant referiert. Und auch wenn der Bezug international vielleicht nicht so explizit ist wie in Deutschland, meine ich doch, dass auch international Kant ein sehr wichtiger Diskussionspartner ist.

ethic@-Wir bedanken uns für das Interview. 